\title{
Application of the Monte Carlo Method for the Estimation of Uncertainty in Radiofrequency Field Spot Measurements
}

\author{
S. Iakovidis, C. Apostolidis, T. Samaras \\ Radiocommunications Laboratory, Department of Physics, Faculty of Sciences, Aristotle University of Thessaloniki, \\ GR-54124 Thessaloniki, Greece, siako@physics.auth.gr
}

\begin{abstract}
The objective of the present work is the application of the Monte Carlo method (GUMS1) for evaluating uncertainty in electromagnetic field measurements and the comparison of the results with the ones obtained using the 'standard' method (GUM). In particular, the two methods are applied in order to evaluate the field measurement uncertainty using a frequency selective radiation meter and the Total Exposure Quotient (TEQ) uncertainty. Comparative results are presented in order to highlight cases where GUMS1 results deviate significantly from the ones obtained using GUM, such as the presence of a non-linear mathematical model connecting the inputs with the output quantity (case of the TEQ model) or the presence of a dominant nonnormal distribution of an input quantity (case of $U$-shaped mismatch uncertainty). The deviation of the results obtained from the two methods can even lead to different decisions regarding the conformance with the exposure reference levels.
\end{abstract}

Keywords: Uncertainty, Monte Carlo, exposure quotient, electromagnetic field measurements, human exposure.

\section{INTRODUCTION}

E XPOSURE TO electromagnetic fields (EMF) is a matter of extended scientific research as well as of public concern, as far as potential health effects are concerned. For this reason, EMF measurements are performed and the results are compared for compliance reasons with respective reference levels determined in exposure guidelines or other legal documents. Radiofrequency EMF exposure levels are evaluated through the measurement of the electric field intensity using frequency selective equipment according to standardized methods [1]. Subsequently, the measurement results are used to evaluate the Total Exposure Quotient (TEQ). The value of the TEQ declares conformity or nonconformity with the specified reference levels (see section 3, below).

Uncertainty is the quantitative tool that expresses the quality of measurement results. Consequently, an uncertainty report must accompany every measurement result, as also specified in [2]. Particularly for EMF measurements, uncertainty estimation is crucial, since it determines conformance or non-conformance with reference levels, thus affecting risk management directly related to human health $[3,4]$.

The first complete guidance which established general rules for evaluating and expressing uncertainty in measurement was given by the "Guide to the Expression of Uncertainty in Measurement" (GUM). Its latest edition [5], which contains minor corrections, is the first edition published under the Charter of the Joint Committee on Guides in Metrology (JCGM). The same year a "Supplement 1 to the 'Guide to the Expression of Uncertainty in Measurement' - Propagation of Distributions using a Monte Carlo Method" [6] was published; in this supplement a numerical technique for evaluating uncertainty based on the Monte Carlo method (GUMS1) is described, which has been applied in several areas other than nonionizing radiation safety $[7,8]$.
In the present work the uncertainty estimation for a frequency selective radiation meter, using the GUMS1, is studied. The results are compared to the ones obtained using the GUM method. The two methods are also applied to the uncertainty estimation of the TEQ in two hypothetic measurement environments (indoors and outdoors).

\section{BRIEF PRESENTATION OF GUM \& GUMS1 METHODS}

\subsection{GUM method}

The theoretical framework described in GUM is based on: a) the law of propagation of uncertainty, and b) the central limit theorem. Consequently, in order to apply correctly the GUM method, the assumptions required for the validity of both a) and b) must be satisfied.

Let $\bar{X}=\left(\mathrm{X}_{1}, \mathrm{X}_{2}, \ldots, \mathrm{X}_{\mathrm{N}}\right)$ denote the input quantities upon which the output quantity $Y$ (measurand) depends and

$$
Y=f(\overline{\mathrm{X}})
$$

the mathematical model linking the inputs to the output. According to the law of propagation of uncertainty (GUM), the combined standard uncertainty of the output quantity will be given by the first order Taylor series expansion of (1):

$$
u_{c}^{2}(y)=\sum_{i=1}^{N}\left(\frac{\partial f}{\partial x_{i}}\right)^{2} u^{2}\left(x_{i}\right)
$$

where $x_{i}$ and $y$ are the best estimates of $\mathrm{X}_{i}$ and $Y$, respectively. The approximation of (2) gives satisfactory results if a) $f$ is linear, and b) $\mathrm{X}_{i}$ are uncorrelated.

The expanded uncertainty $U$ is obtained by multiplying the combined standard uncertainty by a coverage factor $k$ : 


$$
U=k u_{c}(y)
$$

Thus, a coverage interval $[y-U, y+U]$ is specified that encompasses a large fraction of the values of the measurand (e.g. $95 \%$ for $k=1.96$, assuming a normal distribution for the measurand).

According to the central limit theorem, the distribution of the output quantity $Y$ will be approximately normal with expectation

$$
E(Y)=\sum_{i=1}^{N} c_{i} E\left(X_{i}\right)
$$

and variance

$$
\sigma^{2}(Y)=\sum_{i=1}^{N} c_{i}^{2} \sigma^{2}\left(X_{i}\right)
$$

if a) $Y=\sum_{i=1}^{N} c_{i} X_{i}$, b) $X_{i}$ are independent, and c) $\sigma^{2}(Y)$ is much larger than any single component $c_{i}^{2} \sigma^{2}\left(X_{i}\right)$ of a nonnormally distributed $\mathrm{X}_{i}$ [5].

Summarizing the application conditions of both central limit theorem and the law of uncertainty propagation, one can say that: a) If the model is not linear and/or the inputs are not uncorrelated, the central limit theorem and the law of propagation of uncertainty as expressed in (2), do not apply; b) if the model is linear, the input quantities uncorrelated but there is a dominant non-normally distributed input quantity, then the law of propagation of uncertainty applies but the central limit theorem does not.

It is worth mentioning that in case b) the uncertainty estimation is feasible via (2) but not the estimation of the coverage interval limits, since the type of the distribution is not known.

\subsection{GUMS1 method}

The method for uncertainty estimation described in GUMS1 [6], includes the application of the Monte Carlo method (GUMS1) which is based on the 'propagation of distributions'. Utilizing this method, a probability density function (PDF) for the output quantity is obtained which is further used to obtain the expected value for $Y$, its standard uncertainty and the coverage interval for a specified probability.

More specifically, the input quantities are determined, a $\mathrm{PDF}$ is assigned to each one of them, a number of $\mathrm{M}$ samples are drawn from the PDFs for the input quantities, and a number of $\mathrm{M}$ values for $Y$ are calculated using the measurement model (1). The $\mathrm{M}$ values $\left(y_{r}, r=1, \ldots \mathrm{M}\right)$ are used to calculate the expectation $y$ of $Y$ as the average:

$$
y=\frac{1}{M} \sum_{r=1}^{M} y_{r}
$$

and the standard uncertainty $u(y)$ as the standard deviation:

$$
u(y)=\sqrt{\frac{1}{M-1} \sum_{r=1}^{M}\left(y_{r}-y\right)^{2}}
$$

Two different options are suggested by [6] for the coverage interval estimation: a) The probabilistically symmetric coverage interval is calculated by symmetrically subtracting the values from the two distribution limits that do not belong to it, b) The shortest coverage interval is calculated by selecting the shortest of all intervals for the specified coverage probability.

The application of the GUMS1 method is preferable to that of GUM in cases where the model is non-linear or there are one or more dominant non-normally distributed input quantities. However, it requires more computational time compared to GUM.

\section{GUMS1 METHOD APPLIED TO EMF MEASUREMENTS}

In radiofrequency EMF measurements the measured quantity is the electric field intensity, $\mathrm{E}(\mathrm{V} / \mathrm{m})$. The measurements are made using a frequency selective radiation meter, the uncertainty of which depends on the frequency band, as the manufacturer specifies (Table 1.). The conformance with the exposure reference levels is checked via TEQ:

$$
T E Q=\sum_{j=1}^{K} \frac{E_{\text {meas }, j}^{2}}{E_{\text {lim }, j}^{2}}
$$

where $E_{\text {meas }, j}$ is the measured E-field value in the $\mathrm{j}$-th frequency band, $E_{l i m, j}$ the stricter (arithmetically higher) reference level in that frequency band, and $\mathrm{K}$ the number of the frequency bands that are measured. $T E Q<1$ manifests conformity with exposure reference levels, whereas $T E Q>1$ manifests non-conformity.

\subsection{Uncertainty estimation of a frequency selective meter}

The components of uncertainty for different frequency bands were taken from the manufacturer's datasheet. They are included in Table 1. both in linear (\%) and logarithmic $(\mathrm{dB})$ terms.

The GUM and GUMS1 methods were applied with two different considerations, as far as the distribution of the inputs is concerned:

A) Application of manufacturer's distribution type regarding linear terms $(\%)$.

B) Application of manufacturer's distribution type regarding logarithmic terms $(\mathrm{dB})$.

The two considerations above were chosen because they are frequently met in most datasheets or calibration certificates of various manufacturers and calibration laboratories. 
Table 1. Uncertainty estimation results of electric field measurements using frequency selective meter with GUM and GUMS1 methods.

\begin{tabular}{|c|c|c|c|c|c|c|c|c|c|}
\hline & \multirow{3}{*}{\multicolumn{2}{|c|}{$\begin{array}{c}75-300 \mathrm{MHz} \\
\text { Standard uncertainty }\end{array}$}} & & & & & & \\
\hline & & & & \multirow{2}{*}{\multicolumn{2}{|c|}{$\begin{array}{c}900-1400 \mathrm{MHz} \\
\text { Standard uncertainty }\end{array}$}} & \multirow{2}{*}{\multicolumn{2}{|c|}{$\begin{array}{c}1.8-2.2 \mathrm{GHz} \\
\text { Standard uncertainty }\end{array}$}} & \multirow{2}{*}{\multicolumn{2}{|c|}{$\begin{array}{c}2.2-2.7 \mathrm{GHz} \\
\text { Standard uncertainty }\end{array}$}} \\
\hline \multirow{2}{*}{$\begin{array}{l}\text { Components of } \\
\text { Uncertainty }\end{array}$} & \multirow{2}{*}{ Distribution } & & & & & & & & \\
\hline & & $\%$ & $\mathrm{~dB}$ & $\%$ & $\mathrm{~dB}$ & $\%$ & $\mathrm{~dB}$ & $\%$ & $\mathrm{~dB}$ \\
\hline $\begin{array}{l}\text { Uncertainty level of } \\
\text { basic unit }\end{array}$ & Normal & 6.9 & 0.56 & 6.9 & 0.56 & 6.9 & 0.56 & 6.9 & 0.56 \\
\hline $\begin{array}{l}\text { Calibration } \\
\text { uncertainty of } \\
\text { antenna }\end{array}$ & Normal & 6.2 & 0.51 & 9.6 & 0.77 & 6.2 & 0.51 & 6.2 & 0.51 \\
\hline $\begin{array}{l}\text { "Ellipse Ratio" of } \\
\text { antenna }\end{array}$ & Rectangular & 3.4 & 0.29 & 5.6 & 0.46 & 10.9 & 0.87 & 12.9 & 1.01 \\
\hline $\begin{array}{l}\text { Mismatch } \\
\text { uncertainty: basic } \\
\text { unit-antenna } \\
\end{array}$ & U-shape & 10.4 & 0.84 & 8.4 & 0.69 & 5.6 & 0.46 & 6.1 & 0.51 \\
\hline GUM & $95 \%$ & $\begin{array}{l}{[-28.12} \\
+28.12]\end{array}$ & $\begin{array}{l}{[-2.29} \\
+2.29]\end{array}$ & $\begin{array}{l}{[-30.47} \\
+30.47]\end{array}$ & $\begin{array}{l}{[-2.47} \\
+2.47]\end{array}$ & $\begin{array}{l}{[-30.12} \\
+30.12]\end{array}$ & $\begin{array}{l}{[-2.43} \\
+2.43]\end{array}$ & $\begin{array}{l}{[-33.36} \\
+33.36]\end{array}$ & $\begin{array}{l}{[-2.67} \\
+2.67]\end{array}$ \\
\hline GUMS1 (A) & $\begin{array}{l}\text { coverage } \\
\text { interval }\end{array}$ & $\begin{array}{l}{[-25.06} \\
+29.34] \\
\end{array}$ & & $\begin{array}{l}{[-27.68} \\
+32.90]\end{array}$ & & $\begin{array}{l}{[-27.02} \\
+32.03]\end{array}$ & & $\begin{array}{l}{[-29.47} \\
+35.34]\end{array}$ & \\
\hline GUMS1 (B) & & & $\begin{array}{l}{[-2.21} \\
+2.21]\end{array}$ & & $\begin{array}{l}{[-2.45} \\
+2.45]\end{array}$ & & $\begin{array}{l}{[-2.38} \\
+2.38]\end{array}$ & & $\begin{array}{l}{[-2.59} \\
+2.59]\end{array}$ \\
\hline
\end{tabular}

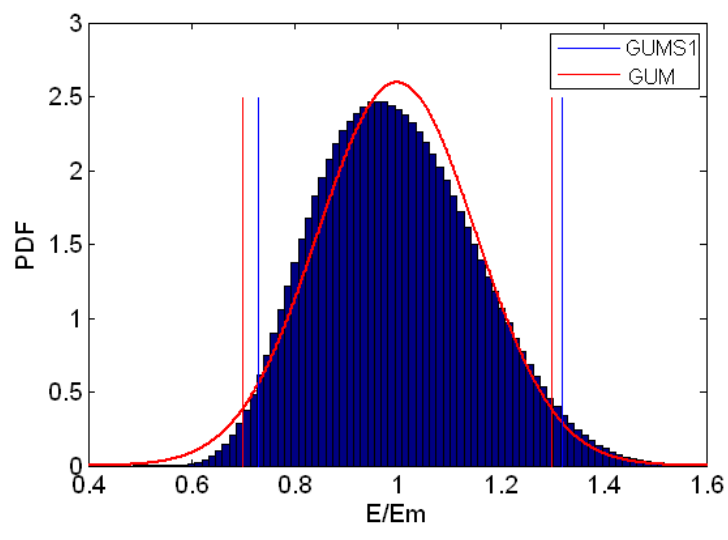

A

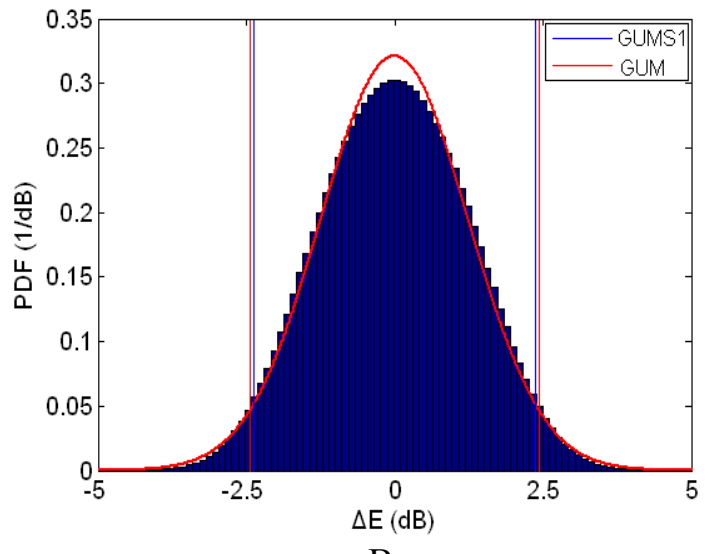

$\mathrm{B}$

Fig.1. Distributions and $95 \%$ coverage interval limits for the output quantity according to GUM (red, line) and GUMS1 (blue, histogram) for the frequency band 1.8-2.2 GHz, for the two different considerations (A and B) described in the text.

The mathematical model linking the inputs to the output is not given by the manufacturer. The multiplicative model was chosen [9],

$$
Y=X_{1} X_{2} X_{3} X_{4}
$$

in case where the inputs are expressed in linear terms (\%) which is equivalent to the additive:

$$
Y=X_{1}+X_{2}+X_{3}+X_{4}
$$

if the inputs are expressed in logarithmic terms (dB).

The uncertainty was evaluated using the two methods (GUM and GUMS1) for both considerations A and B. In order to determine the number of Monte Carlo trials M, the adaptive Monte Carlo method was implemented in MATLAB, [10]. The outcome is that for $M=10^{7}$ the result is correct to two significant decimal digits. The probabilistically symmetric coverage interval was selected instead of the shortest. Due to the probabilistically symmetric coverage interval's symmetry, there is equal probability $(2.5 \%)$ of exceeding either its upper or lower limit. Consequently, it is more appropriate to compare with the interval estimated using GUM, which is also probabilistically symmetric. The results are presented in Table 1., where the limits of the $95 \%$ coverage interval were selected as a means of comparison between the two methods. Fig.1. illustrates the distributions of the output quantity and the coverage interval limits for both methods and for the two different considerations (A and $\mathrm{B}$ ), in the frequency band 1.8$2.2 \mathrm{GHz}$, as an example. 


\subsection{Total Exposure Quotient (TEQ) uncertainty estimation}

The results presented above, and especially those of consideration $\mathrm{A}$, were used for the uncertainty estimation of the $T E Q$ (8) in two different hypothetic measurement environments: indoors and outdoors. The consideration A was selected instead of B, since in the manufacturer's datasheet, the uncertainty estimation using the GUM method is made considering linear terms (\%).

In the indoor environment we considered emissions from DECT cordless telephone and $\mathrm{WiFi}$ transceivers. Consequently, only emissions in the two corresponding frequency bands were considered, i.e. 1.8-2.2 GHz and 2.2$2.7 \mathrm{GHz}$. In the outdoor environment we considered emissions from radio broadcast (FM) and from mobile telephony (GSM, DCS, UMTS). As a result, emissions in the frequency bands $75-300 \mathrm{MHz}, 900-1400 \mathrm{MHz}$, and 1.8$2.2 \mathrm{GHz}$ were considered.
For the choice of the hypothetically measured value two different scenarios were used:

1) $E_{\text {meas }, j}=E_{\text {lim }, j}-2 u_{c, j} E_{\text {lim }, j}$

2) $E_{\text {meas }, j}=E_{\text {lim }, j} / 2$

where $E_{l i m, j}$ is the stricter reference level in the $j$-th frequency band and $u_{c, j}$ the standard combined uncertainty in that band (according to GUM). In other words, in the former scenario the measured field is assumed to have a value that approaches the reference level, whereas in the latter scenario the electric field is only half of the reference level. The adaptive method and a value of $10^{7}$ for $\mathrm{M}$ were used for the calculations, as well. The probabilistically symmetric coverage interval was chosen for the reason mentioned above. The results are presented in Table 2. and Fig.2. and Fig.3.

Table 2. Results of TEQ uncertainty estimation using GUM and GUMS1 methods.

\begin{tabular}{|c|c|c|c|c|c|c|c|c|c|}
\hline \multirow[b]{2}{*}{$\begin{array}{l}\text { Measurement } \\
\text { environment }\end{array}$} & \multirow[b]{2}{*}{ Scenario } & \multicolumn{4}{|c|}{ GUM } & \multicolumn{4}{|c|}{ GUMS1 } \\
\hline & & $T E Q$ & $u(T E Q)$ & $\begin{array}{c}95 \% \\
\text { coverage } \\
\text { interval } \\
\text { lower limit }\end{array}$ & $\begin{array}{c}95 \% \\
\text { coverage } \\
\text { interval } \\
\text { upper limit }\end{array}$ & TEQ & $u(T E Q)$ & $\begin{array}{c}95 \% \\
\text { coverage } \\
\text { interval } \\
\text { lower limit }\end{array}$ & $\begin{array}{c}95 \% \\
\text { coverage } \\
\text { interval } \\
\text { upper limit }\end{array}$ \\
\hline \multirow{2}{*}{ Indoor } & 1 & 0.91 & 0.21 & 0.51 & 1.32 & 0.94 & 0.22 & 0.58 & 1.41 \\
\hline & 2 & 0.50 & 0.11 & 0.28 & 0.72 & 0.51 & 0.12 & 0.31 & 0.77 \\
\hline \multirow{2}{*}{ Outdoor } & 1 & 1.46 & 0.25 & 0.96 & 1.96 & 1.50 & 0.26 & 1.04 & 2.06 \\
\hline & 2 & 0.75 & 0.13 & 0.49 & 1.01 & 0.77 & 0.14 & 0.53 & 1.06 \\
\hline
\end{tabular}

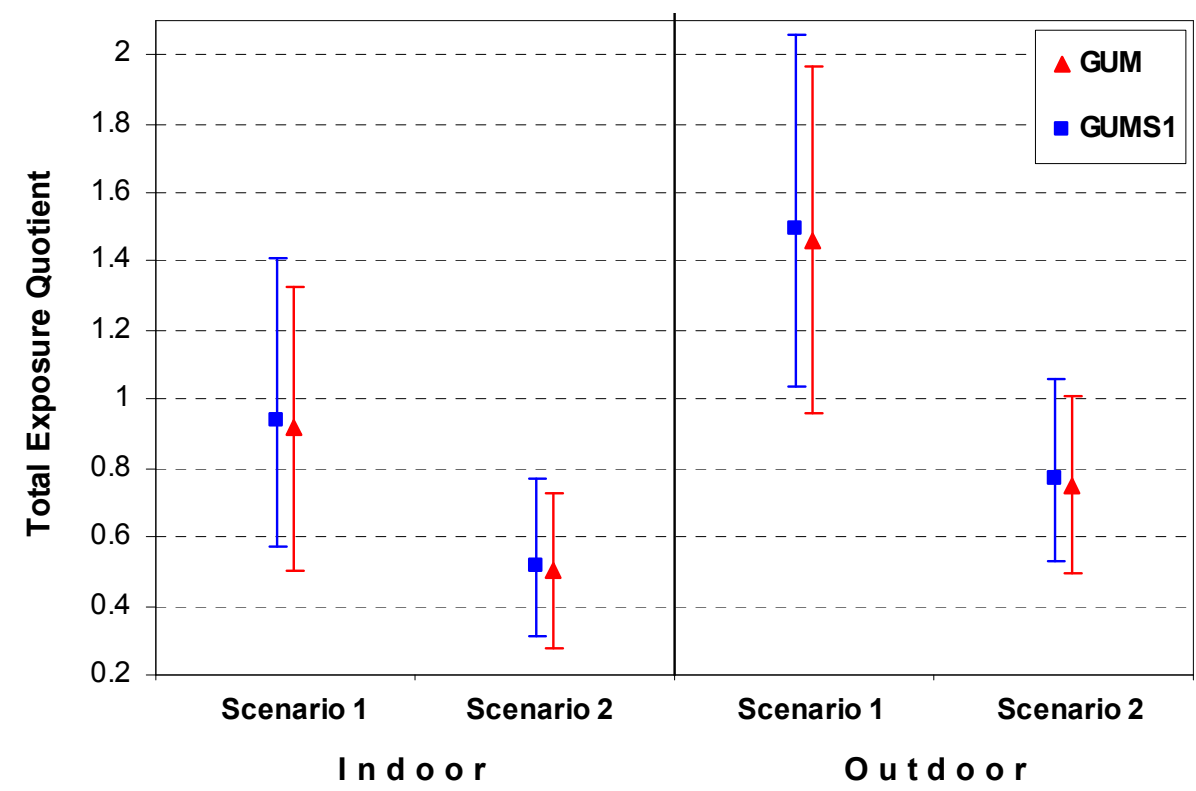

Fig.2. Total Exposure Quotient uncertainty estimation results using GUM and GUMS1 methods. 


\section{DISCUSSION AND CONCLUSIONS}

The application of the GUMS1 method leads to different results compared to GUM for the uncertainty estimation of radiofrequency EMF measurements. This is obvious in cases where either the model is non-linear (subsection 3.1., consideration A and subsection 3.2.), or there is a nonnormally distributed input quantity (subsection 3.1., consideration B).

In particular, the non-linear model (9) which is used in consideration A of subsection 3.1. (considering distributions with linear terms) results in the improper application of both the central limit theorem and the law of propagation of uncertainty (2). Consequently, the output quantity is not normally distributed (Fig.1.A) and the estimated coverage intervals are different.

In the case of consideration B (considering distributions with logarithmic terms, $\mathrm{dB}$ ), the model is linear so the law of propagation of uncertainty applies. On the contrary, due to the non-normal (rectangular) distribution of the ellipse ratio of the antenna, which contributes a significant portion to the combined uncertainty, the central limit theorem does not apply. As a result, the distribution of the output quantity departs from normal and the estimation of the coverage interval limits is different (Fig.1.B). The deviation increases with the increase of the contribution of a non-normally distributed input quantity to the combined uncertainty, as Table 1. illustrates. More specifically, the difference (in dB) between the $95 \%$ coverage interval upper limit as estimated using the two methods, increases from 0.02 to 0.05 and 0.08 $\mathrm{dB}$ as the standard uncertainty of the "Ellipse Ratio" of Antenna (rectangular distribution) increases from 0.46 to 0.87 and $1.01 \mathrm{~dB}$, respectively.

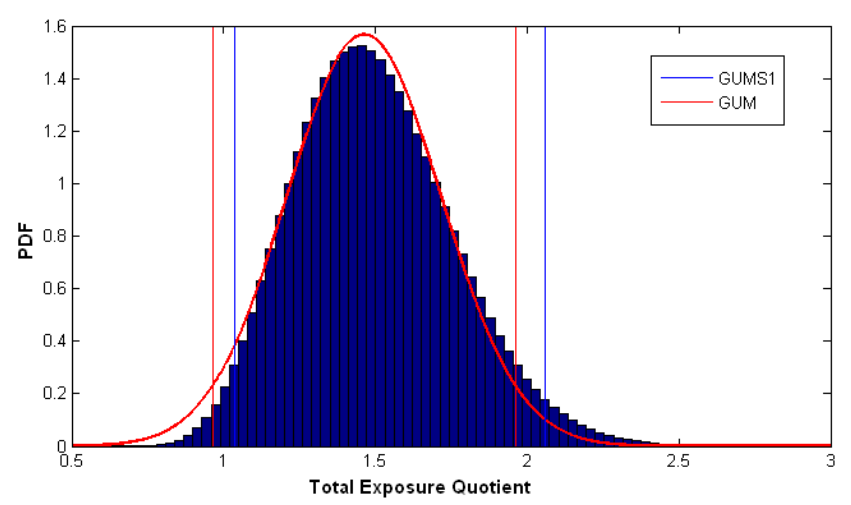

Fig.3. Distributions and $95 \%$ coverage interval limits estimated with GUM (red, line) and GUMS1 (blue, histogram) methods (Outdoor- Scenario 1).

In the cases described in subsection 3.2 the non-linearity of the model used for the $T E Q$ results in different coverage interval estimation using the two methods. This may lead to a different decision as far as conformance with the exposure guidelines is concerned. Particularly, in the case of outdoor measurement, scenario 1, the application of the GUM method results in potential non-conformance only (the lower coverage interval limit is lower than 1) while the application of GUMS1 method results in a definite non-conformance (the whole coverage interval contains values greater than 1). Consequently, the application of GUMS1 method leads to a more accurate estimation of the coverage interval so that, depending on the measurement result, different conclusions could be drawn as far as conformance with exposure guidelines is concerned which is directly related to human health.

\section{REFERENCES}

[1] Hellenic Organisation for Standardization. (2008). Basic standard for the in-situ measurement of electromagnetic field strength related to human exposure in the vicinity of base stations. ELOT EN 50492:2008. Athens.

[2] International Organization for Standardization/ International Electrotechnical Commission. (2005). General requirements for the competence of testing and calibration laboratories. ISO/IEC 17025:2005.

[3] Stratakis, D., Miaoudakis, A., Katsidis, C., Zacharopoulos, V., Xenos, T. (2009). On the uncertainty estimation of electromagnetic field measurements using field sensors: A general approach. Radiation Protection Dosimetry, 133 (4), 240-247.

[4] Vulevic, B., Osmokrovic, P. (2010). Evaluation of uncertainty in the measurement of environmental electromagnetic fields. Radiation Protection Dosimetry, 141 (2), 173-177.

[5] Joint Committee for Guides in Metrology. (2008). Evaluation of measurement data - Guide to the expression of uncertainty in measurement, 1st Edition. JCGM 100:2008.

[6] Joint Committee for Guides in Metrology. (2008). Evaluation of measurement data-Supplement 1 to the 'Guide to the expression of uncertainty in measurement' - Propagation of distributions using a Monte Carlo method, 1st Edition. JCGM 101:2008.

[7] Carobi, C.F.M. (2010). The GUM Supplement 1 and the uncertainty evaluations of EMC measurements. IEEE-EMC Newsletter, 53-57.

[8] Azpurua, M., Tremola, C., Paez, E. (2011). Comparison of the GUM and Monte Carlo methods for the uncertainty estimation in electromagnetic compatibility testing. Progress in Electromagnetics Research B, 34, 125-144.

[9] European Telecommunications Standards Institute. (2001). Electromagnetic compatibility and Radio spectrum Matters (ERM); Uncertainties in the measurement of mobile radio equipment characteristics; Part 1. ETSI TR 100 028-1.

[10] Solaguren-Beascoa Fernandez, M., Alegre Calderon, J.M., Bravo Diez, P.M. (2009). Implementation in MATLAB of the adaptive Monte Carlo method for the evaluation of measurement uncertainties. Accreditation and Quality Assurance, 14 (2), 95-106.

Received November 10, 2014. Accepted April 30, 2015. 